PROCEEDINGS OF THE

AMERICAN MATHEMATICAL SOCIETY

Volume 125, Number 2, February 1997, Pages 329-338

S 0002-9939(97)03532-6

\title{
ON THE CURVES OF CONTACT ON SURFACES IN A PROJECTIVE SPACE. III
}

\author{
M. BORATYNSKI
}

(Communicated by Wolmer V. Vasconcelos)

\begin{abstract}
Suppose a smooth curve $C$ is a set-theoretic complete intersection of two surfaces $F$ and $G$ with the multiplicity of $F$ along $C$ less than or equal to the multiplicity of $G$ along $C$. One obtains a relation between the degrees of $C, F$ and $G$, the genus of $C$, and the multiplicity of $F$ along $C$ in case $F$ has only ordinary singularities. One obtains (in the characteristic zero case) that a nonsingular rational curve of degree 4 in $\mathbf{P}^{3}$ is not settheoretically an intersection of 2 surfaces, provided one of them has at most ordinary singularities. The same result holds for a general nonsingular rational curve of degree $\geq 5$.
\end{abstract}

\section{INTRODUCTION}

In [2] we characterized the smooth curves $C$ which are a set-theoretic complete intersection on a given irreducible surface $F$ in $\mathbf{P}^{3}$ in case $C \not \subset \operatorname{Sing} F$. In [3] the characterization was made more explicit if $C \cap \operatorname{Sing} F$ consists only of rational double points. Moreover we also characterized the curves of contact on $F$ which are not contained in $\operatorname{Sing} F$ provided $F$ has only ordinary singularities (i.e. those which admit a general projection of a nonsingular surface in $\mathbf{P}^{3}$ in the characteristic zero case).

The aim of this paper is to study the smooth curves of contact on $F$ in case $C \subset \operatorname{Sing} F$. A useful tool for this study is the symmetric multiple structures. It turns out that the obvious multiple structure defined on $C$, in case $C$ is a curve of contact on $F$, is symmetric if $\operatorname{Sing} F$ contains at least one pinch point.

Suppose a smooth curve $C$ is a set-theoretic complete intersection of two surfaces $F$ and $G$ with the multiplicity of $F$ along $C$ less than or equal to the multiplicity of $G$ along $C$. One obtains a relation between the degrees of $C, F$ and $G$, the genus of $C$, and the multiplicity of $F$ along $C$ in case the normal cone to $C$ in the scheme defined by $F$ and $G$ is locally (along $C$ ) a complete intersection in the normal bundle to $C$ in $\mathbf{P}^{3}$. This (rather technical) condition is satisfied if $F$ has only ordinary singularities.

Putting together the results of this paper and those of [3] we obtain (in the characteristic zero case) that a nonsingular rational curve of degree 4 in $\mathbf{P}^{3}$ is not

Received by the editors July 25, 1994 and, in revised form, December 13, 1994 and June 27, 1995.

1991 Mathematics Subject Classification. Primary 14H50.

This research was supported by the funds of the Italian Ministry of Education and Scientific Research (MURST).

(C) 1997 American Mathematical Society 
set-theoretically an intersection of 2 surfaces, provided one of them has at most ordinary singularities. The same result holds for a general nonsingular rational curve of degree $\geq 5$.

Of course the main inspiration for this paper is the problem whether any (connected) curve in $\mathbf{P}^{3}$ is a set-theoretic complete intersection. The problem is open even in the case of smooth rational curves. It is known ([6]) that a noncomplete intersection curve cannot be a set-theoretic complete intersection on a nonsingular surface. That is the reason why the singular surfaces come into play in this paper.

\section{Symmetric multiple structures}

In the sequel $C$ will always denote a smooth (connected) curve $\subset \mathbf{P}_{k}^{3}$ ( $k$-algebraically closed) and $I \subset \mathcal{O}_{\mathbf{P}^{3}}$ its ideal sheaf.

Definition. A multiple structure on $C$ is a locally Cohen-Macaulay (lCM) subscheme $\bar{C}$ of $\mathbf{P}^{3}$ with an ideal sheaf $J\left(\mathcal{O}_{\mathbf{P}^{3}} / J\right.$ is locally Cohen-Macaulay) such that $I^{t+1} \subset J \subset I$ for some $t \geq 0$.

For any $i \geq 1$ we define $J_{i}$ as the minimal ideal sheaf containing $J+I^{i}$ which defines a lCM subscheme of $\mathbf{P}^{3}$. So $J_{i}$ is obtained by removing all the embedded components of $J+I^{i}$. We have $J_{1}=I$ and $J_{i}=J$ for $i \geq t+1$ where $t+1$ will denote in the sequel the least $i$ such that $J \supset I^{i}$.

Proposition $1.1([1])$. Let $J \subset \mathcal{O}_{\mathbf{P}^{3}}$ be an ideal sheaf defining a multiple structure on $C \subset \mathbf{P}^{3}$ and let $J_{i}$ be the ideal sheaves defined as before for $i \geq 1$. We put moreover $J_{0}=\mathcal{O}_{\mathbf{P}^{3}}$. Then

$1^{\circ}$. $J_{i} \supset J_{i+1}$ for $i \geq 0$.

$2^{\circ}$. $J_{i} / J_{i+1}$ is a locally free $\mathcal{O}_{C}$-module.

$3^{\circ} . J_{i} J_{j} \subset J_{i+j}$ and the induced map $J_{i} / J_{i+1} \otimes J_{j} / J_{j+1} \rightarrow J_{i+j} / J_{i+j+1}$ is generically surjective.

In the sequel we shall put $E_{i}=J_{i} / J_{i+1}$. In particular $E_{0}=\mathcal{O}_{\mathbf{P}^{3}} / I=\mathcal{O}_{C}$.

Proposition 1.2. Let $\bar{C}$ be a multiple structure on $C$. Then

$$
\operatorname{deg} \bar{C}=\left(\sum_{i=0}^{t} \operatorname{rank} E_{i}\right) \operatorname{deg} C .
$$

Proof. Let us consider the exact sequence

$$
0 \rightarrow E_{i}(n) \rightarrow \mathcal{O}_{\mathbf{P}^{3}} / J_{i+1}(n) \rightarrow \mathcal{O}_{\mathbf{P}^{3}} / J_{i}(n) \rightarrow 0 .
$$

By Riemann-Roch and additivity of the Euler-Poincaré characteristic

$$
\begin{aligned}
& \operatorname{deg} E_{i}+\left(\operatorname{rank} E_{i}\right) \operatorname{deg} C n+\operatorname{rank} E_{i}(1-p(C))+\left(\operatorname{deg} C_{i}\right) n+1-p\left(C_{i}\right) \\
& \quad=\left(\operatorname{deg} C_{i+1}\right) n+1-p\left(C_{i+1}\right)
\end{aligned}
$$

where $C_{i}$ is a (lCM) curve defined by $J_{i}$ and $p\left(C_{i}\right)$ is its (arithmetic) genus. Comparing the terms which contain $n$ we obtain that $\operatorname{deg} C_{i+1}=\operatorname{deg} C_{i}+\left(\operatorname{rank} E_{i}\right) \operatorname{deg} C$. An easy induction completes the proof since $\bar{C}=C_{t+1}$.

Let $\operatorname{Gr}_{I}\left(\mathcal{O}_{\mathbf{P}^{3}}\right)$ denote $\bigoplus_{i \geq 0} I^{i} / I^{i+1}\left(I^{0}=\mathcal{O}_{\mathbf{P}^{3}}\right)$ and for any $J \subset I$ the sheaf of graded ideals $\bigoplus_{i \geq 0}\left(J \cap I^{i}\right)+I^{i+1} / I^{i+1} \subset \operatorname{Gr}_{I}\left(\mathcal{O}_{\mathbf{P}^{3}}\right)$ will be denoted by $J^{*}$ (the sheaf of initial forms of $J$ with respect to the $I$-adic filtration of $\left.\mathcal{O}_{\mathbf{P}^{3}}\right)$. 
Let $J$ define a multiple structure on $C \subset \mathbf{P}^{3}$. Then $I^{i} \subset J_{i}$ for every $i$, so there is a map $I^{i} / I^{i+1} \rightarrow J_{i} / J_{i+1}=E_{i}$ with $\left(J \cap I^{i}\right)+I^{i+1} / I^{i+1}$ contained in its kernel. So we have an induced map $\varphi: \operatorname{Gr}_{I}\left(\mathcal{O}_{\mathbf{P}^{3}}\right) / J^{*} \rightarrow \bigoplus_{0 \leq i \leq t} E_{i}$.

Proposition 1.3. Let $x \in C$. Then the following conditions are equivalent:

$1^{\circ}$. $\varphi_{x}:\left(\operatorname{Gr}_{I}\left(\mathcal{O}_{\mathbf{P}^{3}}\right) / J^{*}\right)_{x} \rightarrow\left(\bigoplus_{0 \leq i \leq t} E_{i}\right)_{x}$ is an isomorphism.

$2^{\circ}$. $\left(\operatorname{Gr}_{I}\left(\mathcal{O}_{\mathbf{P}^{3}}\right) / J^{*}\right)_{x}$ is a (finitely generated) free $\mathcal{O}_{C, x}$-module.

$3^{\circ} .\left(J_{i}\right)_{x}=\left(J+I^{i}\right)_{x}$ for $0 \leq i \leq t$.

$4^{\circ} .\left(\operatorname{Gr}_{I}\left(\mathcal{O}_{\mathbf{P}^{3}}\right) / J^{*}\right)_{x}$ is a Cohen-Macaulay $(C M)$ local ring.

Proof. The implication $1^{\circ} \rightarrow 2^{\circ}$ is obvious. If $2^{\circ}$ holds, then $\left(J+I^{i} / J+I^{i+1}\right)_{x} \approx$ $\left(I^{i} /\left(J \cap I^{i}\right)+I^{i+1}\right)_{x}$ is a free $\mathcal{O}_{C, x^{-}}$-module for $0 \leq i \leq t$. We want to prove that $\mathcal{O}_{\mathbf{P}^{3}, x} /\left(J+I^{i}\right)_{x}$ is $\mathrm{CM}$ for $1 \leq i \leq t$. We induce on $i$. If $i=1$ this is true since $J \subset I$. It is enough to show that $\mathfrak{m}_{x}$-the maximal ideal of $\mathcal{O}_{\mathbf{P}^{3}, x}$-is not associated to $\left(J+I^{i+1}\right)_{x}$ since $\operatorname{dim} \mathcal{O}_{\mathbf{P}^{3}, x} /\left(J+I^{i+1}\right)_{x}=1$. Suppose $\mathfrak{m}_{x} a \in\left(J+I^{i+1}\right)_{x}$ for some $a \in \mathcal{O}_{\mathbf{P}^{3}, x}$. Then $a \in\left(J+I^{i}\right)_{x}$ since $\left(J+I^{i+1}\right)_{x} \subset\left(J+I^{i}\right)_{x}$ and $\mathcal{O}_{\mathbf{P}^{3}, x} /\left(J+I^{i}\right)_{x}$ is CM by the inductive hypothesis. It follows that $a \in\left(J+I^{i+1}\right)_{x}$ since $\left(J+I^{i} / J+I^{i+1}\right)_{x}$ is a free $\mathcal{O}_{C, x}$-module. This proves the implication $2^{\circ} \rightarrow 3^{\circ}$. The implication $3^{\circ} \rightarrow 1^{\circ}$ also holds since

$$
\left(\left(\operatorname{Gr}_{I}\left(\mathcal{O}_{\mathbf{P}^{3}}\right) / J^{*}\right)_{i}\right)_{x}=\left(I^{i} /\left(J \cap I^{i}\right)+I^{i+1}\right)_{x} \approx\left(J+I^{i} / J+I^{i+1}\right)_{x}=\left(E_{i}\right)_{x}
$$

for $0 \leq i \leq t$. Finally the conditions $2^{\circ}$ and $4^{\circ}$ are equivalent since $\left(\operatorname{Gr}_{I}\left(\mathcal{O}_{\mathbf{P}^{3}}\right) / J^{*}\right)_{x}$ is a finite extension of $\mathcal{O}_{\mathbf{P}^{3}, x} / I_{x}$ which is regular.

Remark. The conditions above hold if and only if they hold over the completion of $\mathcal{O}_{C, x}$. Moreover there exists a nonempty open $U \subset C$ such that for $x \in U$ they are satisfied.

Definition. Let $\bar{C}$ be a multiple structure on $C$. Then $\bar{C}$ is called a locally complete intersection (lci) if its ideal sheaf is locally generated by 2 elements.

Definition. Let $\bar{C}$ be a multiple structure on $C$. Then $\bar{C}$ is called symmetric if $\operatorname{rank} E_{i}=\operatorname{rank} E_{t-i}$ for $0 \leq i \leq t$.

Remark. In particular $\operatorname{rank} E_{t}=1$.

Proposition 1.4. Let $\bar{C}$ be a symmetric multiple structure on $C$. Then $\bar{C}$ is a lci if and ony if the pairings $E_{i} \otimes E_{t-i} \rightarrow E_{t}$ (considered in Proposition 1.1) are nonsingular for $0 \leq i \leq t$.

The proof of Proposition 1.4 is the same as the proof of the corresponding statement in case $\operatorname{rank} I / J_{2}=1$ in [4].

Proposition 1.5. Let $\bar{C}$ be a lci multiple structure on $C$. Then the following conditions are equivalent:

$1^{\circ} . \bar{C}$ is symmetric.

$2^{\circ} . J^{*} \subset \operatorname{Gr}_{I}\left(\mathcal{O}_{\mathbf{P}^{3}}\right)$ is generically a complete intersection.

$3^{\circ}$. There exists $x \in C$ such that $\left(J^{*}\right)_{x} \subset \operatorname{Gr}_{I_{x}}\left(\mathcal{O}_{\mathbf{P}^{3}, x}\right)$ is a complete intersection (i.e. $\left(J^{*}\right)_{x}$ is generated by 2 homogeneous elements). 
Proof. Generically $J_{i}=J+I^{i}$ for $0 \leq i \leq t$ since $J_{i}$ is obtained by removing all the embedded components of $J+I^{i}$. Therefore over an open set $U \subset C$

$$
\begin{aligned}
\operatorname{Gr}_{I}\left(\mathcal{O}_{\mathbf{P}^{3}}\right) / J^{*} & =\bigoplus_{0 \leq i \leq t} I^{i} /\left(J \cap I^{i}\right)+I^{i+1} \approx \bigoplus_{0 \leq i \leq t}\left(J+I^{i} / J+I^{i+1}\right) \\
& =\bigoplus_{0 \leq i \leq t} J_{i} / J_{i+1}=\bigoplus_{0 \leq i \leq t} E_{i} .
\end{aligned}
$$

For every $x \in U$, the ideal $\left(J^{*}\right)_{x}$ is a (ht 2) perfect ideal of $\operatorname{Gr}_{I_{x}}\left(\mathcal{O}_{\mathbf{P}^{3}, x}\right)$ since $\operatorname{Gr}_{I_{x}}\left(\mathcal{O}_{\mathbf{P}^{3}, x}\right) /\left(J^{*}\right)_{x}$ is a finite free extension of $\mathcal{O}_{\mathbf{P}^{3}, x} / I_{x}$ which is a discrete valuation ring. Suppose now that $\bar{C}$ is symmetric. It follows from the local version of Proposition 1.4 that the canonical module of $\operatorname{Gr}_{I_{x}}\left(\mathcal{O}_{\mathbf{P}^{3}, x}\right) /\left(J^{*}\right)_{x}$ is free of rank 1 if $x \in U$. By Serre's Lemma $\left(J^{*}\right)_{x}$ is a homomorphic image of a rank 2 projective $\operatorname{Gr}_{I_{x}}\left(\mathcal{O}_{\mathbf{P}^{3}, x}\right)$-module. $\operatorname{Gr}_{I_{x}}\left(\mathcal{O}_{\mathbf{P}^{3}, x}\right)$ is a polynomial ring in 2 variables over a (geometric) discrete valuation ring $\mathcal{O}_{\mathbf{P}^{3}, x} / I_{x}$. So $\left(J^{*}\right)_{x}$ is generated by 2 elements since all the projective $\operatorname{Gr}_{I_{x}}\left(\mathcal{O}_{\mathbf{P}^{3}, x}\right)$-modules are free ([5]). It follows from Nakayama's Lemma that two generators of $\left(J^{*}\right)_{x}$ can be chosen homogeneous since $\left(J^{*}\right)_{x}$ is a homogeneous ideal of $\operatorname{Gr}_{I_{x}}\left(\mathcal{O}_{\mathbf{P}^{3}, x}\right)$. So the implication $1^{\circ} \rightarrow 2^{\circ}$ is proved. $2^{\circ}$ obviously implies $3^{\circ}$. It follows from the proof of $1^{\circ} \rightarrow 2^{\circ}$ that $\operatorname{Gr}_{I}\left(\mathcal{O}_{\mathbf{P}^{3}}\right) / J^{*} \approx \bigoplus_{0 \leq i \leq t} E_{i}$ over a non-empty open subset $U \subset C$. So, for every $0 \leq i \leq t, \operatorname{rank}\left(\operatorname{Gr}_{I}\left(\mathcal{O}_{\mathbf{P}^{3}}\right) / J^{*}\right)_{i}=\operatorname{rank} E_{i}$ where $\left(\operatorname{Gr}_{I}\left(\mathcal{O}_{\mathbf{P}^{3}}\right) / J^{*}\right)_{i}$ denotes the $i$-th homogeneous component of $\operatorname{Gr}_{I}\left(\mathcal{O}_{\mathbf{P}^{3}}\right) / J^{*}$. Let $x \in C$ be such an element that $\left(J^{*}\right)_{x}$ is a complete intersection. Then

$$
\operatorname{rank}\left(\operatorname{Gr}_{I_{x}}\left(\mathcal{O}_{\mathbf{P}^{3}, x}\right) /\left(J^{*}\right)_{x}\right)_{i}=\operatorname{rank}\left(\operatorname{Gr}_{I_{x}}\left(\mathcal{O}_{\mathbf{P}^{3}, x}\right) /\left(J^{*}\right)_{x}\right)_{t-i}
$$

since the Hilbert function of a homogeneous, finite ht 2 complete intersection is symmetric. It follows that $\operatorname{rank} E_{i}=\operatorname{rank} E_{t-i}$ and $\bar{C}$ is symmetric. This proves that $3^{\circ} \rightarrow 1^{\circ}$.

The proof of the implication $1^{\circ} \rightarrow 2^{\circ}$ shows that, for $x \in C,\left(J^{*}\right)_{x}$ is a complete intersection if $\varphi_{x}:\left(\operatorname{Gr}_{I}\left(\mathcal{O}_{\mathbf{P}^{3}}\right) / J^{*}\right)_{x} \rightarrow\left(\bigoplus_{0 \leq i \leq t} E_{i}\right)_{x}$ is an isomorphism. So we obtain the following

Proposition 1.6. Let $\bar{C}$ be a lci symmetric multiple structure on $C$ and let $x \in$ C. If $\varphi_{x}:\left(\operatorname{Gr}_{I}\left(\mathcal{O}_{\mathbf{P}^{3}}\right) / J^{*}\right)_{x} \rightarrow\left(\bigoplus_{0 \leq i \leq t} E_{i}\right)_{x}$ is an isomorphism, then $\left(J^{*}\right)_{x} \subset$ $\operatorname{Gr}_{I_{x}}\left(\mathcal{O}_{\mathbf{P}^{3}, x}\right)$ is a complete intersection.

\section{EASy COMmUtative ALGEBRA}

In the sequel $I$ will denote an ideal of a local regular $\operatorname{ring} R$ with $\operatorname{dim} R=$ 3. Let $f \in R$. We denote by $\operatorname{deg} f$ the largest $s$ such that $f \in I^{s}$ (the degree of $f$ with respect to the $I$-adic filtration of $R$ ). We put $f^{*}=$ the image of $f$ in $I^{\operatorname{deg} f} / I^{\operatorname{deg} f+1} \subset \operatorname{Gr}_{I}(R)=\bigoplus_{i \geq 0} I^{i} / I^{i+1}$ (the initial form of $f$ with respect to the $I$-adic filtration of $R$ ).

Lemma 2.1 ([7]). Suppose $J=(f, g) \subset I \subset R$. If $f^{*}$ and $g^{*}$ form a regular sequence in $\operatorname{Gr}_{I}(R)$, then $J^{*}=\left(f^{*}, g^{*}\right)$ where $J^{*}=\bigoplus_{i \geq 0}\left(J \cap I^{i}\right)+I^{i+1} / I^{i+1} \subset$ $\operatorname{Gr}_{I}(R)$.

Proposition 2.2. Let $I=(x, y) \subset R$ where $x$ and $y$ are the regular parameters of $R$ and $R$ is complete. Suppose that $J=(f, g) \subset I$ where $f$ and $g$ form a regular sequence. If $f^{*} \in \operatorname{Gr}_{I}(R)=(R / I)[X, Y]$ is irreducible $(R / I$ is a discrete valuation ring), then there exists $h \in R$ such that $J=(f, h)$ and $J^{*}=\left(f^{*}, h^{*}\right)$. 
Proof. If the Proposition is not true then it follows from Lemma 2.1 that, for any $h \in R$ such that $J=(f, h), f^{*}$ is a divisor of $h^{*}$ in $\operatorname{Gr}_{I}(R)$. So there exists $r_{1} \in I^{b-a}$ such that $g-r_{1} f \in I^{b+1}$ where $a=\operatorname{deg} f$ and $b=\operatorname{deg} g$. It also follows that $g-r_{1} f-r_{2} f \in I^{b+2}$ for some $r_{2} \in I^{b-a+1}$ since $J=\left(f, g-r_{1} f\right)$. In this way we obtain a sequence of elements $r_{1}, r_{2}, \ldots, r_{i}, \ldots$ such that $r_{i} \in I^{b-a+i}$ and, for every $i, g-\left(r_{1} f+r_{2} f+\cdots+r_{i} f\right) \in I^{b+i}$. Since $R$ is complete, $\sum r_{i} \in R$ and $g=\left(\sum r_{i}\right) f$. But this is impossible since $f$ and $g$ form a regular sequence.

Proposition 2.3. Let $I=(X, Y) \subset k[[X, Y, Z]]$ and suppose $J=(X Y, g)$ such that $J \supset I^{i}$ for some $g \in I$ and $i \geq 1$. Then

$1^{\circ} . J=\left(X Y, \alpha X^{k}+\beta Y^{l}\right)$ with $\alpha, \beta$ invertible $\in k[[X, Y, Z]], k, l \geq 1$.

$2^{\circ}$. For all $i \geq 1, k[[X, Y, Z]] /\left(J+I^{i}\right)$ is Cohen-Macaulay.

Proof. Let $J=(X Y, g)$ such that $J \supset I^{i}$ for some $g \in I$ and $i \geq 1$. Then $g$ $\bmod Y=\alpha X^{k}$ with $\alpha \in k[[X, Z]]$ invertible and $k \geq 1$. It follows that $g=\alpha X^{k}+r Y$ for some $r \in k[[X, Y, Z]] . g \bmod X=\beta Y^{l}$ with $\beta \in k[[Y, Z]]$ invertible and $l \geq 1$. So we obtain that $(r \bmod X) Y=\beta Y^{l}$ and $r=\beta Y^{l-1}+s X$ for some $s \in k[[X, Y, Z]]$. We infer that $g=\alpha X^{k}+\beta Y^{l}+s X Y$ and $J=\left(X Y, \alpha X^{k}+\beta Y^{l}\right)$. This proves $1^{\circ}$.

In order to prove $2^{\circ}$ because of the symmetry of $X$ and $Y$, we can suppose that $k \leq l$. We obviously have $J+I=I$. Moreover $J+I^{i}=\left(X Y, X^{i}, Y^{i}\right)$ if $2 \leq i \leq k$, $\left(X Y, X^{k}, Y^{i}\right)$ if $k+1 \leq i \leq l$ and $J+I^{i}=J$ for $i \geq l+1$. It is easy to see that the ideals $J+I^{i}$ are determinantal and therefore, for all $i \geq 1, k[[X, Y, Z]] /\left(J+I^{i}\right)$ is Cohen-Macaulay.

Remark. The multiple structure defined by $J$ on $\operatorname{Spec} k[[X, Y, Z]] / I$ is symmetric only if $k=l$.

Proposition 2.4. Let $I=(X, Y) \subset k[[X, Y, Z]]$. Then there does not exist $J=$ $(X Y Z, g)$ with $g \in I$ such that $J \supset I^{i}$ for some $i \geq 1$.

Proof. It suffices to note that $J \bmod Z$ is principal whereas $I \bmod Z$ is a height 2 ideal.

\section{Multiple struCtures Defined By two surfaces}

Suppose $C=\operatorname{supp}(F \cap G)$ where $F$ and $G$ are two surfaces in $\mathbf{P}^{3}$. In the sequel $J$ will denote the ideal sheaf corresponding to the ideal of the homogeneous coordinate ring of $\mathbf{P}^{3}$ which is generated by the equations of $F$ and $G$. Obviously $J$ defines a multiple structure on $C$.

Proposition 3.1. For the multiple structure $\bar{C}$ defined above $E_{t} \approx \omega_{C}(4-m-n)$ where $m=\operatorname{deg} F, n=\operatorname{deg} G$ and $\omega_{C}$ is a canonical bundle on $C$.

Proof. The exact sequence $0 \rightarrow E_{t} \rightarrow \mathcal{O} / J_{t+1} \rightarrow \mathcal{O} / J_{t} \rightarrow 0\left(\mathcal{O}=\mathcal{O}_{\mathbf{P}^{3}}\right)$ induces the map $\omega_{\bar{C}} \approx \operatorname{Ext}^{2}\left(\mathcal{O} / J_{t+1}, \omega_{\mathbf{P}^{3}}\right) \rightarrow \underline{\operatorname{Ext}}^{2}\left(E_{t}, \omega_{\mathbf{P}^{3}}\right)$ which is surjective since $\mathcal{O} / J_{t}$

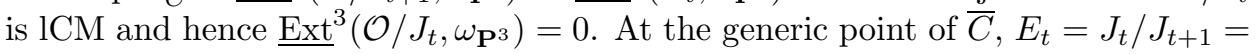
$J_{t} / J$ is the highest nonvanishing power of the maximal ideal of the corresponding local ring. $J_{t} / J_{t+1}$ is generically generated by one element since the local ring of $\bar{C}$ at its generic point is Gorenstein. It follows that $\operatorname{rank} E_{t}=\operatorname{rank} J_{t} / J_{t+1}=1$. 
We obtain $\underline{\operatorname{Ext}}^{2}\left(E_{t}, \omega_{\mathbf{P}^{3}}\right) \approx \omega_{\bar{C}} \otimes \mathcal{O}_{C} \approx \mathcal{O}_{C}(m+n-4)$ since also $\underline{\operatorname{Ext}}^{2}\left(E_{t}, \omega_{\mathbf{P}^{3}}\right)$ is a rank one locally free sheaf on $C$. We further obtain

$$
\begin{aligned}
E_{t} & \approx \underline{\operatorname{Ext}}^{2}\left(\underline{\operatorname{Ext}}^{2}\left(E_{t}, \omega_{\mathbf{P}^{3}}\right), \omega_{\mathbf{P}^{3}}\right) \approx \underline{\operatorname{Ext}}^{2}\left(\mathcal{O}_{C}(m+n-4), \omega_{\mathbf{P}^{3}}\right) \\
& \approx \underline{\operatorname{Ext}}^{2}\left(\mathcal{O}_{C}, \omega_{\mathbf{P}^{3}}\right)(4-m-n) \approx \omega_{C}(4-m-n)
\end{aligned}
$$

which was to be proved.

For any $x \in C$ let $f_{x} \in I_{x} \subset \mathcal{O}_{\mathbf{P}^{3}, x}$ be the element corresponding to the equation of $F$. We denote by $\operatorname{deg} f_{x}$ and $f_{x}^{*}$ respectively the degree and the initial form of $f_{x}$ with respect to the $\widehat{I}_{x}$-adic filtration of $\widehat{\mathcal{O}}_{\mathbf{P}^{3}, x}$. Note that $\operatorname{deg} f_{x}=$ degree of $f_{x}$ with respect to the $I_{x}$-adic filtation of $\mathcal{O}_{\mathbf{P}^{3}, x}$. In the same way we define $\operatorname{deg} g_{x}$ and $g_{x}^{*}$ where $g_{x}$ is the element of $I_{x}$ corresponding to $G$.

Let $F \subset \mathbf{P}^{3}$ be a surface containing $C$. Then $F \in H^{0}\left(I^{k}(m)\right)$ where $m=$ $\operatorname{deg} F$ and $k \geq 1$ (note a slight abuse of the notation). So $F$ induces a section of $I^{k} / I^{k+1}(m)$. Note that there exists a unique $k$ such that the induced section of $I^{k} / I^{k+1}(m)$ is nonzero.

Theorem 3.2. Suppose $C=\operatorname{supp}(F \cap G)$ with $\operatorname{deg} F=m$ and $\operatorname{deg} G=n$ and suppose that, for every $x \in C, J_{x}^{*} \subset \operatorname{Gr}_{I_{x}}\left(\mathcal{O}_{\mathbf{P}^{3}, x}\right)$ is a complete intersection. If $F$ defines a nonzero section of $I^{k} / I^{k+1}(m)$ and $G$ defines a nonzero section of $\left.I^{l} / I^{l+1}(n)\right)$ with $k \leq l$, then

$1^{\circ}$. $m n=k(t-k+2) d$ where $d=\operatorname{deg} C$.

$2^{\circ} . \omega^{\otimes k(t-k+2)} \approx \mathcal{O}_{C}(k n+(t-k+2)(m-4 k))$ where $\omega$ is a canonical bundle of $C$.

In particular $k(t-k+2)(2 g-2)=d[k n+(t-k+2)(m-4 k)]$ where $g$ denotes the genus of $C$.

Proof. Let $x \in C$. Then $J_{x}^{*}=\left(h_{1}, h_{2}\right)$ with $k=\operatorname{deg} h_{1} \leq \operatorname{deg} h_{2}$. Moreover the Hilbert function of $\bigoplus_{0 \leq i \leq t} E_{i}$ is equal to the Hilbert function of

$$
\operatorname{Gr}_{I_{x}}\left(\mathcal{O}_{\mathbf{P}^{3}, x}\right) / J_{x}^{*}=\left(\mathcal{O}_{\mathbf{P}^{3}, x} / I_{x}\right)[X, Y] /\left(h_{1} h_{2}\right)
$$

since $\varphi: \operatorname{Gr}_{I}\left(\mathcal{O}_{\mathbf{P}^{3}}\right) / J^{*} \rightarrow \bigoplus_{0 \leq i \leq t} E_{i}$ is an isomorphism by Proposition 1.3.

$$
\operatorname{rank} E_{i}= \begin{cases}i+1, & 0 \leq i \leq k-1, \\ k, & k \leq i \leq \operatorname{deg} h_{2}-1, \\ k+\operatorname{deg} h_{2}-i-1, & \operatorname{deg} h_{2} \leq i \leq t .\end{cases}
$$

It follows that $\operatorname{deg} h_{2}=t-k+2$ since $\operatorname{rank} E_{t}=1$. An easy calculation shows that $\sum_{i=0}^{t} \operatorname{rank} E_{i}=k(t-k+2)$. To prove $1^{\circ}$ it suffices to apply Proposition 1.1 to the multiple structure $\bar{C}$ and note that $\operatorname{deg} \bar{C}=m n$ (Bezout).

Suppose first that $k=\operatorname{deg} h_{1}<\operatorname{deg} h_{2}$. Then, for each $x \in C, J_{x}^{*}$ is generated by $f_{x}^{*}$ with $\operatorname{deg} f_{x}^{*}=k$ and some element of $\operatorname{Gr}_{I_{x}}\left(\mathcal{O}_{\mathbf{P}^{3}, x}\right)$ of degree $t-k+2>k$. It follows that $J_{x}^{*}$ in degree $t-k+1$ is generated by $f_{x}^{*} . F$ induces a monomorphism $\mathcal{O}_{C}(-m) \rightarrow I^{k} / I^{k+1}$. We obtain that

$$
J_{t-k+1}^{*} \approx \mathcal{O}_{C}(-m) \otimes I^{t-2 k+1} / I^{t-2 k+2}
$$

and

$$
E_{t-k+1} \approx S^{t-k+1}(N) / \mathcal{O}_{C}(-m) \otimes S^{t-2 k+1}(N)
$$


where $N$ denotes the conormal bundle $I / I^{2}$ and $S^{i}(N)$ its $i$-th symmetric power. It follows from Proposition 1.4 and Proposition 3.1 ( $\bar{C}$ is obviously symmetric) that

$$
E_{k-1} \approx \underline{\operatorname{Hom}}\left(E_{t-k+1}, \omega(4-m-n)\right) \approx\left(E_{t-k+1}\right)^{*} \otimes \omega(4-m-n) .
$$

So we get

$$
S^{k-1}(N) \approx\left(S^{t-k+1}(N) / \mathcal{O}_{C}(-m) \otimes S^{t-2 k+1}(N)\right)^{*} \otimes \omega(4-m-n)
$$

since $E_{k-1} \approx S^{k-1}(N)$. Extracting the highest exterior powers we obtain that

$$
\begin{aligned}
\left(\omega^{\otimes-1}(-4)\right)^{\otimes k(k-1) / 2} \approx & \mathcal{O}_{C}(-m)^{\otimes t-2 k+2} \otimes\left(\omega^{\otimes-1}(-4)\right)^{\otimes(t-2 k+1)(t-2 k+2) / 2} \\
& \otimes\left(\omega^{\otimes-1}(-4)\right)^{\otimes-(t-k+1)(t-k+2) / 2} \otimes(\omega(4-m-n))^{\otimes k}
\end{aligned}
$$

since $\Lambda^{2} N \approx \omega^{\otimes-1}(-4)$ and, for any $i, \Lambda^{i+1} S^{i}(N) \approx\left(\Lambda^{2} N\right)^{\otimes i(i+1) / 2}$ (apply the splitting principle). Now an easy (but tedious) calculation concludes the proof.

If $k=\operatorname{deg} h_{2}$, then $t=2 k-2$ and $E_{k-1} \approx \underline{\operatorname{Hom}}\left(E_{k-1}, \omega(4-m-n)\right) . E_{k-1}=$ $S^{k-1}(N)$ and proceeding as above we obtain $2^{\circ}$ with $t=2 k-2$.

Extracting the degrees of both sides of $2^{\circ}$ we easily obtain that

$$
k(t-k+2)(2 g-2)=d[k n+(t-k+2)(m-4 k)] .
$$

Proposition 3.3. Let $J$ be the ideal sheaf of the multiple structure on $C=$ $\operatorname{supp}(F \cap G)$ which was defined above. Suppose that $\varphi: \operatorname{Gr}_{I}\left(\mathcal{O}_{\mathbf{P}^{3}}\right) / J^{*} \rightarrow \bigoplus_{0 \leq i<t} E_{i}$ is an isomorphism over a (nonempty) open set $U \subsetneq C$. If for all $x \in C-\bar{U}$ either $f_{x}^{*} \in \operatorname{Gr}_{\widehat{I}_{x}}\left(\widehat{\mathcal{O}}_{\mathbf{P}^{3}, x}\right)$ or $g_{x}^{*} \in \operatorname{Gr}_{\widehat{I}_{x}}\left(\widehat{\mathcal{O}}_{\mathbf{P}^{3}, x}\right)$ is irreducible, then, for every $x \in C$, $J_{x}^{*} \subset \operatorname{Gr}_{I_{x}}\left(\mathcal{O}_{\mathbf{P}^{3}, x}\right)$ is a complete intersection.

Proof. By Proposition 2.2 the extension of $J_{x}^{*}$ to $\operatorname{Gr}_{\widehat{I}_{x}}\left(\widehat{\mathcal{O}}_{\mathbf{P}^{3}, x}\right)$ is a complete intersection if $x \in C-U$. It follows that also $J_{x}^{*} \subset \operatorname{Gr}_{I_{x}}\left(\mathcal{O}_{\mathbf{P}^{3}, x}\right)$ is a complete intersection. By Proposition 1.5 the multiple structure $\bar{C}$ is symmetric. Applying Proposition 1.6 we obtain that $J_{x}^{*} \subset \operatorname{Gr}_{I_{x}}\left(\mathcal{O}_{\mathbf{P}^{3}, x}\right)$ is a complete intersection if $x \in U$.

Corollary 3.4. Suppose $C=\operatorname{supp}(F \cap G)$ with $\operatorname{deg} F=m$ and $\operatorname{deg} G=n$ and let the ideal sheaf $J$ of the multiple structure $\bar{C}$ satisfy the hypotheses of Proposition 3.3, i.e. there exists a nonempty open set $U \subsetneq C$ such that $\varphi_{x}:\left(\operatorname{Gr}_{I}\left(\mathcal{O}_{\mathbf{P}^{3}}\right) / J^{*}\right)_{x} \rightarrow$ $\left(\bigoplus_{0 \leq i \leq t} E_{i}\right)_{x}$ is an isomorphism for $x \in U$ and for all $x \in C-U$ either $f_{x}^{*} \in$ $\operatorname{Gr}_{\widehat{I}_{x}}\left(\widehat{\mathcal{O}}_{\mathbf{P}^{3}, x}\right)$ is irreducible or $g_{x}^{*} \in \operatorname{Gr}_{\widehat{I}_{x}}\left(\widehat{\mathcal{O}}_{\mathbf{P}^{3}, x}\right)$ is irreducible. If $F$ defines a nonzero section of $I^{k} / I^{k+1}(m)$ and $G$ defines a nonzero section of $\left.I^{l} / I^{l+1}(n)\right)$ with $k \leq l$, then

$1^{\circ}$. $m n=k(t-k+2) d$ where $d=\operatorname{deg} C$.

$2^{\circ} . \omega^{\otimes k(t-k+2)} \approx \mathcal{O}_{C}(k n+(t-k+2)(m-4 k))$ where $\omega$ is a canonical bundle of $C$.

In particular $k(t-k+2)(2 g-2)=d[k n+(t-k+2)(m-4 k)]$ where $g$ denotes the genus of $C$.

Remark. In view of the Remark following Proposition 1.3 the condition which concerns the points of $C-U$ is the only essential hypothesis. 


\section{ORdinARY SINGULARITIES}

Recall that a surface $F \subset \mathbf{P}_{k}^{3}(\operatorname{ch} k=0)$ admits ordinary singularities if $\operatorname{Sing} F$ is a curve (possibly reducible) and, for $x \in \operatorname{Sing} F, \widehat{\mathcal{O}}_{F, x}$ is one of the following:

1. For almost all $x \in \operatorname{Sing} F, \widehat{\mathcal{O}}_{F, x} \approx k[[X, Y, Z]] /(X Y)$ is an ordinary double point.

2. $\widehat{\mathcal{O}}_{F, x} \approx k[[X, Y, Z]] /(X Y Z)$ is an ordinary triple point.

3. $\widehat{\mathcal{O}}_{F, x} \approx k[[X, Y, Z]] /\left(X^{2}-Y^{2} Z\right)$ is a pinch point.

It is well known that if $\operatorname{ch} k=0$, then a generic projection of any (projective) smooth surface into $\mathbf{P}_{k}^{3}$ admits only ordinary singularities.

Theorem 4.1. Let $C$ be a (smooth) curve contained in the singular locus of a surface $F \subset \mathbf{P}^{3}$ which along $C$ admits only ordinary singularities and among them at least one pinch point. If there exists a surface $G \subset \mathbf{P}^{3}$ such that $C=\operatorname{supp}(F \cap G)$, then

$$
\begin{aligned}
& m n=2 t d \text { and } \\
& \omega^{\otimes 2 t} \approx \mathcal{O}_{C}(2 n+t m-8 t) \text { if } G \text { is singular along (whole) } C \text { or } \\
& m n=(t+1) d \text { and } \\
& \omega^{\otimes(t+1)} \approx \mathcal{O}_{C}(m+(t+1)(n-4)) \text { otherwise }
\end{aligned}
$$

where as before $\omega$ is a canonical bundle of $C, m=\operatorname{deg} F, n=\operatorname{deg} G, d=\operatorname{deg} C$ and $t$ is the least $i$ such that $J \supset I^{i+1}$ ( $I$ is the ideal sheaf of $C$ and $J$ is the ideal sheaf corresponding to the ideal generated by $F$ and $G$ ).

Proof. Let $U$ denote the (open) set of $C$ which consists of ordinary double points of $F$. It follows from Proposition 1.3 and Proposition 2.3 that $\varphi_{x}:\left(\operatorname{Gr}_{I}\left(\mathcal{O}_{\mathbf{P}^{3}}\right) / J^{*}\right)_{x} \rightarrow$ $\left(\bigoplus_{0<i<t} E_{i}\right)_{x}$ is an isomorphism for $x \in U$. Moreover it follows from Proposition 2.4 that $F$ has no ordinary triple points along $C$. So if $x \in C-U$, then

$$
f_{x}^{*}=X^{2}-Y^{2} Z \in \operatorname{Gr}_{\widehat{I}_{x}}\left(\widehat{\mathcal{O}}_{\mathbf{P}^{3}, x}\right)=k[[Z]][X, Y]
$$

is irreducible. The application of Theorem 3.2 with $k=2$ and $k=1$ respectively concludes the proof. (Note that in case $k=1$ the roles of $m$ and $n$ are interchanged.)

Proposition 4.2. Let $C$ be a (smooth) curve contained in the singular locus of a surface $F \subset \mathbf{P}^{3}$. If $F$ admits along $C$ only ordinary double points, then $\omega^{\otimes 2} \approx$ $\mathcal{O}_{C}(2 m-8)$ where $m=\operatorname{deg} F$.

Remark. Note that we do not make any $C=\operatorname{supp}(F \cap G)$ assumption!

Proof. Recall first that for any locally free sheaf $P$ there is a map $\varphi: S^{2}\left(P^{*}\right) \rightarrow$ $\left(S^{2} P\right)^{*}$ defined locally by $\varphi\left(f_{1} \otimes f_{2}\right)(x \otimes y)=f_{1}(x) f_{2}(y)+f_{1}(y) f_{2}(x)$ with $f_{1}, f_{2} \in$ $P^{*}$ and $x, y \in P$.

The surface $F$ defines the map $\mathcal{O}_{C}(-m) \rightarrow I^{2} / I^{3}=S^{2}\left(I / I^{2}\right)$. Composing the dual map $S^{2}\left(I / I^{2}\right)^{*} \rightarrow \mathcal{O}_{C}(m)$ with $\varphi$ we obtain $\alpha: S^{2}\left(\left(I / I^{2}\right)^{*}\right) \rightarrow \mathcal{O}_{C}(m)$. We claim that the induced map $\alpha^{\prime}:\left(I / I^{2}\right)^{*} \rightarrow \underline{\operatorname{Hom}}\left(\left(I / I^{2}\right)^{*}, \mathcal{O}_{C}(m)\right)$ is an isomorphism. It suffices to check this at the completion of the local ring at each point of $C$. So we can assume that $I=(x, y)$ and $\mathcal{O}_{C}(-m)$ is freely generated by one element $e$ (say). Moreover the map $\mathcal{O}_{C}(-m) \rightarrow I^{2} / I^{3}$ associates $x y$ to $e$. (Note a slight abuse of notation). Let $\left(x^{*}, y^{*}\right)$ be the dual basis of the basis $(x, y)$ of $I / I^{2}$. One checks easily that $\alpha\left(\left(x^{*}\right)^{2}\right)=0, \alpha\left(x^{*} y^{*}\right)=e$ and $\alpha\left(\left(y^{*}\right)^{2}\right)=0$. It follows that $\alpha^{\prime}$ is an isomorphism. So we obtain that

$$
\left(I / I^{2}\right)^{*} \approx \underline{\operatorname{Hom}}\left(\left(I / I^{2}\right)^{*}, \mathcal{O}_{C}(m)\right) \approx\left(I / I^{2}\right) \otimes \mathcal{O}_{C}(m) .
$$


Hence

$$
\left(\Lambda^{2}\left(I / I^{2}\right)\right)^{*} \approx \Lambda^{2}\left(I / I^{2}\right) \otimes \mathcal{O}_{C}(2 m)
$$

and

$$
\left(\omega^{\otimes-1}(-4)\right)^{*} \approx \omega^{\otimes-1}(-4) \otimes \mathcal{O}_{C}(2 m) .
$$

This implies that $\omega^{\otimes 2} \approx \mathcal{O}_{C}(2 m-8)$ which was to be proved.

Corollary 4.3. Let $C$ be a smooth rational curve of degree $d \geq 3$ contained in the singular locus of a surface $F \subset \mathbf{P}^{3}$. Then $C$ is not a set theoretic intersection on $F$ if $F$ has along $C$ ordinary singularities.

Proof. Suppose $F$ has along $C$ only ordinary double points. Then by Proposition 4.2 $\omega^{\otimes 2} \approx \mathcal{O}_{C}(2 m-8)$. Extracting degrees we obtain $-4=d(2 m-8)$. This is not possible if $d \geq 3$, so $F$ admits along $C$ at least one pinch point and we can apply Theorem 4.1. If the singular locus of $G$ contains $C$, then $m n=2 t d$ and extracting degrees we obtain $-4 t=d(2 n+m t-8 t)$. Putting $n=2 t d / m$ in the last equation we get (after simplifications) the following quadratic equation with respect to $m$ :

$$
d t m^{2}+4 t(1-2 d) m+4 t d^{2}=0 .
$$

So the discriminant $D=16 t^{2}(1-2 d)^{2}-16 t^{2} d^{3} \geq 0$. We infer that $(1-2 d)^{2}-d^{3} \geq 0$. It follows that $d^{2}-3 d+1 \leq 0$ since $(1-2 d)^{2}-d^{3}=-(d-1)\left(d^{2}-3 d+1\right)$ and $d \geq 3$. If $d^{2}-3 d+1 \leq 0$ holds, then $d \leq(3+\sqrt{5}) / 2<3$. So if $C$ is set-theretically the intersection of $F$ and $G$, then $C$ is not contained in the singular locus of $G$. So by Theorem $4.1 \omega^{\otimes(t+1)} \approx \mathcal{O}_{C}(m+(t+1)(n-4))$. Taking degrees we obtain $-2(t+1)=d[m+(t+1)(n-4)]$. It follows that $n<4$ since $-2(t+1)<0$, $d, m, t+1>0$. The cases $n=1$ and $n=2$ are not possible since $C$ is not a plane curve and $C$ is not a set-theoretic complete intersection on a quadric. If $n=3$ we obtain $-2(t+1)=d m-d(t+1)$. But by Theorem $4.1 d(t+1)=3 m$. So $-2(t+1)=(d-3) m$. This equality cannot hold since $d \geq 3$. It follows that $C$ is not a set theoretic complete intersection on $F$.

Corollary 4.4. Let $C$ be a smooth rational curve on a surface $F \subset \mathbf{P}_{k}^{3}$ which admits only ordinary singularities. If $\operatorname{deg} C=4$ or $\operatorname{deg} C \geq 5$ and $C$ is general, then $C$ is not a set theoretic complete intersection on $F$.

Proof. By [3] $C$ is not a set theoretic complete intersection on $F$ if $C \not \subset \operatorname{Sing} F$ (the assumption that $F$ is irreducible is not used in the proof). If $C \subset \operatorname{Sing} F$ we apply Corollary 4.3 .

Remark. Generality of $C$ means that its normal bundle in $\mathbf{P}^{3}$ is semistable.

\section{ACKNOWLEDGMENT}

This paper owes a great deal to the splendid work of the referee whose criticism contributed enormously to its present form.

\section{REFERENCES}

[1] C. Banica, O. Forster, Multiplicity structures on space curves, The Lefschetz centennial conference, part I, Contemp. Math., vol. 58, Amer. Math. Soc., Providence, RI, 1986, pp. 47-64. MR 88C: 32018

[2] M. Boratynski, On the curves of contact on surfaces in a projective space, Algebraic $K$ theory, Commutative Algebra and Algebraic Geometry, Contemp. Math., vol. 126, Amer. Math. Soc., Providence, RI, 1992, 1-8. MR 92m:14033 
[3] M. Boratynski, On the curves of contact on surfaces in a projective space II, Rend. Sem. Mat. Univers. Politecn. Torino, Vol. 48. 4 (1990), 439-455. MR 94j:14031

[4] M. Boratynki, Locally complete intersection multiple structures on smooth algebraic curves, Proc. Amer. Math. Soc. 115, (1992), 877-879. MR 92J:14039

[5] H. Lindel, On projective modules over polynomial rings over regular rings, Algebraic $K-$ theory, Lecture Notes in Math; vol. 966, Springer-Verlag, Berlin, Heidelberg, New York, 1982, pp. 169-179. MR 84d:13009

[6] L. Robbiano, A problem of complete intersections, Nagoya Math. J. 52, (1973) 129-132. MR 48:11132

[7] P. Valabrega, G. Valla, Form rings and regular sequences, Nagoya Math. J. Vol. 72 (1978), 93-101. MR 80d:14010

Dipartimento di Matematica, via E Orabona 4, 70125 Bari, Italy

E-mail address: boratyn@pascal.dm.uniba.it 